\title{
Fault detection of broken rotor bar in LS-PMSM using random forests
}

\begin{abstract}
This paper proposes a new approach to diagnose broken rotor bar failure in a line startpermanent magnet synchronous motor (LS-PMSM) using random forests. The transient current signal during the motor startup was acquired from a healthy motor and a faulty motor with a broken rotor bar fault. We extracted 13 statistical time domain features from the startup transient current signal, and used these features to train and test a random forest to determine whether the motor was operating under normal or faulty conditions. For feature selection, we used the feature importances from the random forest to reduce the number of features to two features. The results showed that the random forest classifies the motor condition as healthy or faulty with an accuracy of $98.8 \%$ using all features and with an accuracy of $98.4 \%$ by using only the mean-index and impulsion features. The performance of the random forest was compared with a decision tree, Naïve Bayes classifier, logistic regression, linear ridge, and a support vector machine, with the random forest consistently having a higher accuracy than the other algorithms. The proposed approach can be used in industry for online monitoring and fault diagnostic of LS-PMSM motors and the results can be helpful for the establishment of preventive maintenance plans in factories.
\end{abstract}

Keyword: Line start-permanent magnet motor; Broken rotor bar; Fault detection; Startup current; Statistical features; Random forest 